\title{
Treinta y tres años de cirugía correctora en tetralogía de Fallot
}

\author{
Osvaldo Artaza B. ${ }^{1}$; Stephan Haecker D. ${ }^{1}$; Oscar Gómez V. ${ }^{1}$; \\ Claudio Arretz V. ${ }^{1}$; Luis León M. ${ }^{1}$
}

\section{Surgical results in patients with tetralogy of Fallot}

\begin{abstract}
From january 31, 1957 throughout december 31. 1989, 591 patients undenvent intracardiac repair for tetralogy of Fallot (ToF), -exciuding patients with pulmonary atresia- at our cardiovascular center, Santiago, Chile. Global lethatity results were analized in two separate eartier periods between years 195 ? and 1967 (n. 92); 1968 through. out 1979 ( $\mathrm{n}$ : 243) and in 256 children operated on from 1980 to 1989 postoperatory gvents and follow up were aiso analized as to age at operation, surgical technique and results. Global lethality decreased from $32.6 \%$ in the first decade to $16.9 \%$ in the second one and $4.29 \%$ in the iast periog 1198019891 . There were no deaths among patients who underwent primary repair before 12 months of age ( $n$ : 17 ) in the last decade. Neither differences in lethalıty rates among patients treated by different surgical techniques (infundibular patch, trans-annular patch, trans-atrial approach. septal ciefect closure with vaivulotomy alone\}. Low cardiac output in the postoperative period was the first cause of death (82\% such cases). and was mainly associated with older age and persistence of high right ventricular pressure $1>0.85$ of left ventricular pressure). After 57.5 months mean follow up, actual grade 1 functional capacity (NYHA] was found in $98.8 \%$ patients operated on the last period. A worldwide tendency towards early primary repair of Tof is now recognized, with low operative lethality. Further prospective studies are needed in order to evaluate the long rerm outcome of the different surgical techniques actualty employed.
\end{abstract}

iKey words: Tetralogy of Fallot, surgical repair, morbidity, lethatity.)

La tetralogía de Fallot (TF) (comunicación interventricular, estenosis pulmonar, hipertrofia del ventrículo derecho $y$ dextroposición aórti. ca) fue descrita hace más de 100 affos ${ }^{1}$, su frecuencia entre las cardiopatías congénitas es aproximadamente $10 \% 2$ e incluye un amplio espectro de anomaljas, según si la obstrucción del tracto de salida (ts) de ventrículo derecho(VD)es de predominio infundibular, valvular, supravalvular o mixto, y si hay otras alteraciones congénitas o adquiridas (cortocircuitos sistémico-pulmonares paliativos previos) de las arterias pulmonares u otras malformaciones cardiacas mayores aso. ciadas ${ }^{3}$.

Después de la printera corrección efectuada con buen éxito, por Lillehei el año 1954 en la Universidad de Minnesota ${ }^{4}$, se inició en nuestro pais, en 1957, la corrección a corazón abierto de esta malformación. Desde entonces se han desarrollado múltiples avances en los métodos

1. Servicio Cardiovastular, Hospilal "Luis Calvo Mackenna. quirúrgicos y cuidados perioperatorios, destacando las técricas de protección miocárdica (cardioplejia), el abordaje transouricular (con el fin de minimizar la ventriculotomía), distintas alternativas de ampliación del tsVD y la tendencia a hacer correcciones primarias cada vez más precoces $^{5-12}$.

En nuestro centro la reparación primaria electiva de los pacientes oligosintomáticos se ha hecho tradicionalmente después de $\operatorname{los} 2$ años de edad ${ }^{3,6}$, pero casos seleccionados, con anatomía favorable (diametro adecuado) de las ramas pulmonares, se operaban antes del año de vida. Cuando habia hipoxemia severa o crisis anoxémicas que no cedian a propanolol y la anatomía pulmonar no aconsejaban la corrección en una etapa ${ }^{13}$, se hacía una operación paliativa previa (creaciôn de un cortocircuito sistémicopulmonar, conectando la arteria subclavia contralateral de donde desciende el cayado aórtico, con la arteria pulmonar respectiva -operación de Blalock clásica-.. o interponiendo un tubo protésico entre las arterias subclavia y pulmonar) y la corrección definitiva en una etapa posterior. 
Los factores de riesgo en la reparación de la TF son la hipoplasia de las ramas pulmonares ${ }^{14}$, agenesia de velos pulmonares's; necesidad de efectuar parche transanular ${ }^{14,16}$, edad menor ${ }^{14}$ alta concentración de hemoglobina (hipoxia crónica importante) y presión igual o mayor que la sistémica en el ventriculo derecho que en el izquierdo (Vl) -en relación de 0,85 o mayordespués de la reparación ${ }^{17}$. La letalidad global de la reparación en la TF varía entre 4 y $8 \%$ en otras experiencias ${ }^{17,18}$, que en las corregidas con parche transanular varia entre 4 y $23 \% 19,20$. Con la que todos los pacientes evolucionan con insuficiencia pulmonar leve o moderada que podría traducirse en morbilidad al largo plazo ${ }^{18}$. La obstrucción residual tracto de salida, comunicación interventricular residual, arritmias ven. triculares, alteraciones de la conducción e insuficiencia pulmonar severa pueden producir malos resultados alejados ${ }^{21,22}$. El bloqueo completo de rama derecha (BCRD), ocurre en la mayoría de las TF operadas; se han encontrado ectopias ventriculares hasta en $25 \%$ de los pacientes. Las alteraciones severas del ritmo pueden explicar los casos alejados de muerte súbita ${ }^{23}$. En $4 \%$ de los casos se señala la necesidad de reoperaciones por lesiones residuales, cuya leta. lidad no sería mayor que en la operación inicial ${ }^{2}$

El propósito de este análisis es describir una experiencia nacional con el tratamiento quirúrgico de esta malformación, en términos de letalidad operatoria, en distintos períodos y en el último decenio, así como la evolución postoperatoria, según diferentes factores de riesgo y variaciones de las técnicas quirúrgicas empleadas.

\section{Pacientes y Métodos}

Se revisaron los antecedentes de los 591 pacientes con tetralogia de Fallot (se excluyeron los casos de atresia pulnonar con comunicación interventricular) operados en el Servicio Cardiovascular del Hospital "Luis Calvo Mackenna", entre el 31 de enero de 1957 (fecha de la primera intervención) y el 31 de diciembre de 1989). La experiencia acumulada se separó en tres períodos: I: años 1957 a 1967 ; II: años 1968 a 1979 , y J[l: años 1980 a 1989 . Para los 2 primeros períodos se analiza la letalidad en genetal, mientras para el último los resultados quirúrgicos, las complicaciones postoperatorias y resultados del seguimiento según edad, variables perioperatorias (hemoglobina preoperatoria, cirugía paliativa previa, tiempo de circulación extracorpórea, tiempo de pinzamiento aórtico ( $t$ 'Ao), presiones de ventrículo derecho e izquierdo (VD/VI) inmediatamente después de la reparación, también se compararon los resultados según la técnica quirúrgica empleada, que consistió en ventriculotom ía $y$ ampliación del infundíbuto con parche (p, inf) en 134 pacientes $(52 \%)$; Abordaje por vía transauricular derecha (AD) en 23 pacientes $(9 \%)$; Ventriculotomía y parthe transanular (PTA) en 86 pacientes (33\%) $y$, finalmente, cierre del defecto interventricular y sólo valyulotomia (Val) en 13 pacientes $(5 \%)$. Fn el primer período (1957-1967) se operaton 92 pacientes, 48 de sexo masculino $(52 \%)$. Edad promedio: $?$ añas 9 meses márgenes 1 a 20 años, peso promedio: $22,4 \mathrm{~kg}$. No se elgió a ningún paciente antes de un año de edad. En todos el abordaje fue por ventriculotomía y no se efectuó ningún parche transanular (tabla 1). In el segundo período (1968-1979) se intervinieron 243 pacientes, $136(56 \%)$ varones, su edad promedio era $\mathcal{S}$ años 4 meses, márgenes 5 meses a 19 años, peso promedio: $16,8 \mathrm{~kg}$. En esta serie se corrigieron 9 pacientes menotes de un año. La vía de abordaje fue por ventriculotomía. En 79 se efectuó parche transanular y en 34 (14\%) se habia hecho cirugía paliativa previa. En el tercer periodo $(1980-1989)$ se opetaron 256 pacientes: $138(54 \%)$ de sexo masculino, edad promedio 4 años 5 meses +43 meses, márgenes 3 meses a 19 años, peso $16,3 \pm 8,9 \mathrm{~kg} ; 17$ eran menores de un año $(6,6 \%)$ (tabla 1). En 47 casos $(18,4 \%)$ se habia hecho un corto-

Tabla 1

Edad y sexo de 591 pacientes operados de tetralogía de Fallot. según período de estudio

\begin{tabular}{lccc}
\hline & $1957-1967$ & $1968-1979$ & $1980-1989$ \\
\hline Pacientes & 92 & 243 & 256 \\
Edad promedio & $7 \mathrm{a} 9 \mathrm{~m}$ & $5 \mathrm{a} 4 \mathrm{~m}$ & $435 \mathrm{~m}$ \\
1 año & - & $9(3,7 \%)$ & $17(6,6 \%)$ \\
Sexo masculino & $48(52,0 \%)$ & $136(56,0 \%)$ & $138(54,0 \%)$ \\
\hline
\end{tabular}


Tabla 2

Letalidad general $y$ en pacjentes menores de un año entre 591 niños operados de tetralogía de liallot en diferentes períodos

\begin{tabular}{lcccccc}
\hline Pet ído & Pacientes & $\begin{array}{c}\text { Letalidad general } \\
\text { Fallecidos }\end{array}$ & $\%$ & Pacientes & $\begin{array}{c}\text { Letalidad }<1 \text { ano } \\
\text { Fallecidos }\end{array}$ & $\%$ \\
\hline $1957-1967$ & 92 & 30 & 32,60 & - & - & - \\
$1968-1979$ & 243 & 41 & 16,87 & 9 & 3 & 33 \\
$1980-1989$ & 256 & 11 & 4,29 & 17 & 0 & 0 \\
\hline
\end{tabular}

circuitu sistétnico-pulmonar previo; 76 pacientes $[29,7$ por cjentol recibieron propanolol en el período preoperatorio para prevenir crisis anoxémicas. La hemoylobina en el períndo preoperatorio inmediato era $16,3 \pm 2,9 \mathrm{~g} / \mathrm{dl}$. Tres de estos pacientes tonian TF con agenesia de los velos valvulates pulmonares. No se encontraton diferencias significativas de edad, operaciones precedentes paliativas $y$ cifras preoperatorias de hemoglobina. Al agrupar a los pacientes según las técnicas quirúrgicas empleadas entre los menores de un año (promedio: $7,4 \pm 3,5$ iлeses, peso $6,8=2.9 \mathrm{~kg}$ ) $41,2 \%$ recibieron propanolol preoperatorio, en ninguno se realkaton operaciones paliativas previas $y$ la concentracion de hemoglobina fue menor $(14 \pm 4,1 \mathrm{~g} / \mathrm{dl}$ que en los ortos grupos de edad. En este grupo 2 se corrigieron por $\mathrm{AD}, 6$ con $\mathrm{P}$. inf y 9 con $\mathrm{PT} A$.

Desde 1957 se han hecho varias innovaciones en las técnicas quirúrgicas. Las estándar del último período son esternotomía media y circulación extracorpórea con oxigenador de burbuja en todos los casos. In los lactantes menores se utilizó hipotermia profunda $116^{\circ} \mathrm{C}$ y paro cardiaco hipotérnico. En los demás pacientes se usó circulación extracorpórea con hipotermia moderada (hasta $25^{\circ} \mathrm{C}$ ). Antes del año sólo se efectuaba protección miocárdica con hipotermia, desde ese afio se inició técnica de cardioplejía cristaloidea a $40 \mathrm{C}(20 \mathrm{ml} \cdot \mathrm{kg})$ y desde 1988 ésta si realiza cada 20 minutos, enfriando el pericardio cun solución fría en administración continua. Antes de iniciar la citculación extracurpórea se disecan los cortocircuitos sistémicopulmonares pretios y se ligan una vez comenzada ésta. Para jniciar la reparación intracardíaca se pinza la aorta y se descomprimen las cavidades izquierdas.

Hasta el aìo 1988 la rcparación intracasdíaca consistió en hacer ventriculotomia sobre el infundíbulo, proIongándola hacia el tronco de la arteria pulmonas sj el anillo fuese hipoplásico. Luego se resecaba el infundíbulo hipertrófico y octúa el defecto septal interventrjeular con un parche de materiai sintético (Dacrón ${ }^{\circ}$ ). Unid vez cerradn ol defecto interventricular, en caso de estenosis valvular pulmonar, se realizaba valvulotomia bajo vision directa desde el ventrículo o el tronco de la arteria pulmotaar. Se calibraba el anillo valvular pulmo. nar según tablas de pacificos $y$ se evaluaba la necesidad de ampliar con un parche transanular, que se hacía inicialmente con pericardio nativo, más tarde con pericardio fijado en glutaraldehido o politetrafluoetileno (PTFE). La ventriculotomía se cerraba on un parche de PTFE, hubiese o no sido necesario uno transanular. Desde el año 1988 , cuando no hab ía hipoplasia uniforme del infundíbulo, se usó como alternativa la reparación por la via transauricular derecha, usando una auricujotomia derecha amplia y a través de la vátuula tricúspide se reseca el infundíbulo y se cierra el defecto interventricular. Si la infundibulectomía es insuficiente, se completa por vía 1ransarterial pulmonar. Se incinde longitudinalmente el tronco de la arteria pulmonar, se efectúa la valvulotomia $y$ se completa la resección infundibular cuando éstas son necesarias. Si queda estrecho el anillo valvulas se prolonga la arteriotomía 1 a $2 \mathrm{~cm}$ hacja el infundíbulo (ventriculotomía mínima $)^{10}$, se suelca la pinza de la aorta $y$ durante el recalentamiento se cierran la auriculotom ía y la arteriotomía. Esta última, en caso de ventriculotom í mínima, se cierra mediante parche de pericardio fijado. Actualmente en todos los pacientes se vierra el defecto interventricular por esta vía, lo que permite hacer ventriculotomías más pequerias. Cuando es necesario usar parche transanular $y$ es posible que quede insuficiencia pulmonar importante, se utiliza una válvala monocúspide de pericardio. Cuando la calibración de las arterias pulmonares confirma estenosis, éstas se amplían con un parche de pericardio. En algunos casos, sïn estenosis infundibular, con anillo pulmonar adecuado. sólo se efectuó cierre del defecto interventricular $y$ valuulotomia. Siempre se instala cable epicárdico de tnarcapaso y catéteres auriculares. Lna vez desconectado el paciente de la circulación extracorpórea y 30 minutos después de neutralizar la heparina con protamina, se toman las presiones ventriculares por puncion directa.

\section{Resultados}

En el primer periodo la letalidad global fue de $32,6 \%$ (30 de 92 pacientes). En el segundo (1968-1979) se redujo a 41 de 243 pacientes $(16,9 \%)$, tres entre los nueve menores de un año fallecieron y 17 de $79(22 \%)$ nifíos corregidos con parche transanular.

Los resultados que siguen se refieren al período más reciente (1980-89). En él se intervinieron 256 pacientes y fallecieron sólo $11(4,29$ 
por ciento), ninguno entre los 17 menores de 1 año (tabla 2). La letalidad fue también menor en los niños mayores de tres años: dos en 145 $(1,4 \%), p<0,01$. El tiempo de circulación extracorpórea promedio fue de $57,3 \pm 13,7 \mathrm{~min} y$ el de pinzamiento aórtico $30,1 \pm 10,2 \mathrm{~min}$. Las relaciones de las presiones de ventriculares post. operatorias (PVD/PVI) fueron $>0,85$ en 10,93 por ciento de los casos, 0,5 a 0,85 en $66,02 \%$ $y<0,5$ en $23,05 \%$ de los pacientes. No hubo diferencias significativas según edad, t'CEC, t'Ao, nivel de hemoglobina, cirugia paliativa previa y la técnica quirúrgica empleada, siendo la mortalidad de los pacientes corregidos con parche transanular de $3 / 86,3,5 \%$. En este perído no se registró mortalidad tard́a, tampoco mortalidad en pacientes con agenesia de los velos pulmonares. En los nifios con presión de VD en niveles sistémicos se observó una relación significativa $(\mathrm{p}<0,01)$, con bajo débito postoperatorio y muerte (tabla 3). El postoperatorio inmediato fue nomal (definido como extubación traqueal antes de 24 horas, ausencia de bajo débito severo o moderado y de complicaciones o compromiso de otros órganos o sistemas $^{24}$, en $71,9 \%$. En conjunto, el problema más frecuente fue el bajo débito moderado o severo (n: $31,12,1 \%$, tabla 4), cuya incidencia se relacionó directa $y$ significativamente con la edad (no ocurrió en menores de un año) y la letalidad, pues $82 \%$ de los fallecidos tuvieron bajo débito $y$, a la inversa, murieron 9 de 31 $\{29 \%)$ de los niños con débito bajo y sólo 2 de $225(0,8 \%)$ con débito normal $(\mathrm{p}<0,01)$. Se encontrason relaciones significativas $(p>0,05)$ entre bajo debito y concentración de hemoglobina ( 84 pacientes, $32,8 \%$, tenian hemoglobina sobre $18 \mathrm{~g} / \mathrm{dl}$ y $20,2 \%$ de éstos tuvieron débito bajo), también con uso previo de propanolol, ya que 76 pacientes, $29,7 \%$ recibieron propanolol preoperatorio, $18(23,7 \%)$ de estus tuvieron bajo débito y $7(9,2 \%$, p: NS) fallecieron. Las reoperaciones fueron necesarias en 12 pacientes, tres precoces en niños reparados con parche infundibular (una para hemostasia, una con CEC por defecto interventricular residual y gradiente severo de presión en el tsVD, otra para retirar drenaje). En los pacientes tratados con parche transanular hubo cuatro reoperaciones precoces por hemostasia y cuatro tardías (una con CEC por estenosis infundibular, otra también con CEC para resecar diafragma subaórti$\infty$, una para retirar un cuerpo extraño - alambre- $y$ otra con CEC para suturar de un defecto interventricular recanalizado). En el grupo tratado con cierre del defecto interventricular y sólo valvulotomía hubo una reoperación precoz con CEC para cerrar una comunicación interventricular residual.

En 235 de los 245 pacientes vivos del tercer período $(96 \%)$, se pudo efectuar un seguimiento confiable, por un promedio de 56 meses $\pm 39,8$ meses, siendo menor para el grupo abordado por $A D$ (promedio de 16 meses). En 98,8\% de los pacientes la capacidad funcional es 1 , independientemente de la técrica empleada y la edad. Se pesquisó bloqueo completo de la rama derecha del haz de Hiss (BCRD) $38,4 \%$ e incompleto (BIRD) en 30,6\%, no encontrándose diferencias según las técricas. En dos pacientes operados con

Tabla 3

Relación del cuocicnte de presión (Ventrículo derecho/ventr j́culo izquierdo) con bajo débito postuperatorio y letalidad en 256 pacientes operados de tetralogía de Fallot entre 1980 y 1989

\begin{tabular}{lrrrr}
\hline & $\mathrm{n}$ & Con bajo débito & Fallecidos \\
\hline VD/VI $>0,85$ & 28 & $10(35,7 \%)$ & $4(14,3 \%)$ \\
VD/VI $0,5-0,85$ & 169 & $19(11,2 \%)$ & $6(3,6 \%)$ \\
VD/VI $<0,50$ & 59 & $2(3,4 \%)$ & $1(1,7 \%)$ \\
Total & 256 & $31(12,1 \%)$ & $11(4,3 \%)$ \\
\hline
\end{tabular}

VD: presión en ventrículo derecho.

VI : presión en ventrículo izquietdo. 
Tabla 4

Complicaciones postoperatorias en 256 pacientes corregidos de tetralogía de Fallot según técnica empleada

\begin{tabular}{|c|c|c|c|c|c|c|}
\hline & P. Inf. & AD & PTA & Val. & Total & $\boldsymbol{q}_{\mathrm{s}}$ \\
\hline Bajo débito & 18 & 2 & 11 & 2 & 31 & 12,1 \\
\hline Bloqueo $\mathrm{AVt}^{1}$ & 15 & 2 & 9 & - & 26 & 10,2 \\
\hline Falla respiratoria ${ }^{2}$ & 11 & 1 & 7 & - & 19 & 7,4 \\
\hline Infección porroperatorja ${ }^{3}$ & 5 & 2 & 6 & - & 13 & 5,0 \\
\hline Insuticiencia renal & 5 & - & 2 & - & 7 & 2,7 \\
\hline (peritoneodiúlisis) & (3) & & (2) & & (5) & \\
\hline Falla de bemostasia & 1 & - & 4 & 1 & 6 & 2,3 \\
\hline Hemorragia digesti & 4 & - & 1 & . & 5 & 1,9 \\
\hline Distunción neurológica & 3 & - & 1 & - & 4 & 1,6 \\
\hline Coagulopatía & 1 & - & 3 & - & 4 & \\
\hline Hemotórax & 3 & - & 1 & - & 4 & \\
\hline Derrame pleural & 1 & - & 3 & - & 4 & \\
\hline Síndrome postpericardiostomía & 3 & - & 1 & - & 4 & \\
\hline Atelectasia masiva & - & - & 3 & -- & 3 & \\
\hline Traquenstom $\mathfrak{i}^{\mathrm{a}}$ & 2 & - & 1 & - & 3 & \\
\hline Arritmias scyeras s & 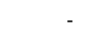 & 2 & 1 & - & 3 & \\
\hline Insutīiencia hepática & 1 & -. & 1 & - & 2 & \\
\hline Estenusis subglótica & 2 & - & - & - & 2 & \\
\hline Hemorragia pulmonar & 1 & & - & - & 1 & \\
\hline Nelimotórax & - & 1 & - & - & 1 & \\
\hline
\end{tabular}

Bloquto AV transitorio del postoperatorio inmediato.

Necesidades de ventilación mecánica $>72$ h por cavsa pulmonar.

Infecciones int rahospitalarias severas.

Necesidad de Traqucostom ia por entu bación traqueal prolotigada.

- Dos casos de taquicardia paroxística supraventricular y uno de fibrílación ventricular.

parche infundibular se presentaron arritmias (extrasistoles ventriculares aislados frecuentes) que han sido estudiados con prueba de esfuerzo y registro electrocardiografico continuo (Holter). En $15,3 \%$ de los casos se detectó soplo sistólico residual y soplo diastólico suave de insuficiencia pulmonar leve en $59,2 \%$, sin diferencias entre las técnicas empleadas. La morbilidad entre los pacicntes tratados con parche transanular no fue significativamente mayor que en los otros grupos. Hubo cuatro estenosis de ts VD, dos aneurismas del parche (en ambos casos había usado parche de pericardio nativo sin fijarlo con glutaraldehido). Un paciente tratado con PTA tiene insuficiencia pulmonar importante (según clínica $y$ seguimiento ecocardiográfico) y permanece en capacidad funcional II. Las lesiones residuales que no requirieron reoperación han sido detectadas en 13 pacientes $(5,3 \%)$. Entre los niños reparados con PTA hubo 3 casos de estenosis pulmonar moderadas (una del infundíbulo $y$ del origen de la arteria pulmonar derecha, una en el origen de la arteria pulmonar izquierda $y$ otro con estenosis leve de arteria pulmonar de- recha), dos pacientes con aneurisma moderado del parche transanular (en éstos se utilizó pericardio), dos casos de CIV residual pequeña y un paciente con insuficiencia pulmonar severa. En el grupo en que se usó parche infundibular, 5 pacientes presentaron $\mathrm{CIV}$ residual pequeña. El seguimiento ha sido exclusivamente clínico en $79,6 \%$ de los pacientes, con controles ecográficos en 50 niños, cateterísmo cardíaco postoperatorio en 10 , electrocardiograma continuo (Holter) y prueba de esfuerzo en dos. Cuatro pacientes tienen secuelas (dos casos con hemiparesia, en rehabilitación, y 2 niños con pectus carinatum postesternotomia).

\section{Discusión}

Desde el primer período a la fecha se aprecia clara tendencia a correcciones más precoces $y$ sostenida disminución de la letalidad, hasta jlegat a niveles excelentes en relación a experiencias extranjeras. En nuestra casuística, la letalidad de los pacientes tratados con PTA no es diferen- 
te que en los restantes, siendo ella más alta en muchos otros centros ${ }^{17-20}$. Esta positiva disminución de la mortalidad se puede deber a una multitud de variables difíciles de evaluar, como mejor conocimiento de la patología, avances en las técnicas quirúrgicas (especialmente en el área de la protección miocárdica) y en los cuida. dos postoperatorios inmediatos.

Como factores de riesgo concordamos con las publicaciones citadas en relación a la presión de VD mayor o igual a la sistemica. No pudimos establecer una relación clara entre concentración elevada de hemoglobina $>18 \mathrm{~g} / \mathrm{dl}$ ), bajo débito y letalidad, tampoco entre estos últimos y uso previo de propanolol, lo que en nuestro medio es más difícil de analizar, ya que por limitación de recursos en muchos pacientes su empleo es más bien debido a la necesidad de retardar la intervención operatoria. Los mayores de 3 años tienen significativa menor mortalidad y ésta no se registró el último período en los menores de un año, si bien cabe sefialar que en este grupo los pacientes fueron seleccionados por sus condiciones anatómicas muy favorables. Los pacientes con agenesia de velos fueron muy pocus como para establecer grado de riesgo en ellos.

E] débito cardíaco bajo, moderado o severo, fue el más importante de los problemas postoperatorios y la principal causa de muerte. El porcentaje de reoperaciones fue bajo $(4,9 \%)$, también el de lesiones residuales. La capacidad funcional es excelente, pero el seguimiento es aún insuficiente e incompleto para obtener conclusiones más finas sobre función del ventrículo derecho, el riesgo de aparición de arritmias importantes y morbilidad asociada al parche transanular a largo plazo. Es de destacar el alto porcentaje de seguimiento que obtuvimos en estos pacientes, lo que desgraciadamente no se repite en otros casos en que ocurren altos porcentajes de deserción, para lo que no tenemos una explicación clara. La evaluación postoperatoria alejada es fundamentalmente clinica, debido a que la alta presión asistencial de nuestro servicio nos limita la disponibilidad de otros instrumentos, to que nos resta valiosa información de la evolución a largo plazo. Aún así, la revisión de esta experiencia nos ha motivado a realizar estudios más completos en ese sentido, en los pacientes que llevan ya más de 10 años, desde su corrección quirúrgica. EI breve plazo transcurrido desde que se inició la téc- nica transauricular en nuestro servicio no nos permite aún conclusiones. Sin duda, a pesar de los avances, aún no está todo dicho en relación a la reparación de la tetralogía de Fallot.

\section{Resumen}

En 1957 se inició en el Centro Cardiovascular del Hospital "Luis Calvo Mackenna" la corrección quirúrgica de la tetralogía de Fallot (excluidos los niños con atresia pulmonar y comunicación interventricular). Desde entonces hasta diciembre de 1989 se han intervenido 591 de estos pacientes. Se revisa la letalidad entre los 92 y 243 pacientes operados desde 1957 a 1967 y desde 1968 a 1979 , respectivamente, asi como en los 256 pacientes del periodo 1980 a 1989 , en los que tambièn se analizan las complicaciones postoperatorias y los resultados a corto y mediano plazo (seguimiento promedio 57,5 meșes), según edad y técnica quirúrgica emplea. da. La letalidad general ha descendido de 32,6\% en el primer período a $4,29 \%$ entre los años 1980 y 1989, en que no fallecieron pacientes corre. gidos primariamente antes del año de vida (17 casos). No hubo diferencias significativas de letalidad según técnicas empleadas (parche infundibular, parche transanular, vía transanular 0 valvulotomía exchusiva). La principal causa de muerte fue bajo débito cardíaco en el periodo postoperatorio (82\%). En $98,8 \%$ de los pacientes del último periodo de estudio la capacidad funcional actual es I (NYHA). Sólo un paciente tiene evidencia de insuficiencia severa de la válvula puimonar. Existe una clara tendencia a hacer reparaciones más precoces y notoria disminución de la letalidad, requiriéndose mayores estudios prospectivos de largo plazo, para evaluar morbilidad futura de las distintas técnjcas actualmente empleadas.

(Palabras claves: Tetralogia de Fallot, tratamiento quirúrgico, complicaciones, letalidad.)

\section{Referencias}

1. Fallot $A$ : Contribution a L'anatomie pathologique de la maladie blue (cyanose cardiaque). Mat Med 1988; $25: 77-93$.

2. Pinsky W' Arcinieges E. Tetralogy of Fallot. Pediatr Cin North Am 1990; 37 : 179-192.

3. Zillenielo $R$ : Cardiopatías Cianóticas. En Manual de Cardiología y Cirugía Cardiovascular Infantil. Artaza O y Zilleruclo R. Ed. Mediterráneo. Santiago, Chile 1989: 51-74. 
4. Lillehei $C W$, Cohen $M$, Warden $H E$ : Direct vision intracardiac surgical correction of the tetralogy of Fallot, pentalogy of Fallot, and pulntonary atresia defects. Report of first ten case. Ann Surg $1955 ; 142: 418-421$.

5. Pacifico AD, Kirklin JW, Blackstone EH: Surgical, management of pulmonary stenosis in tetralogy of Fallot. I Thorac Cardiovase Surg 1977; 74: $582 \cdot 595$,

6. Gómez $O$ : Cirugía en las cardiopatías congénitas cianóticas. En Manual de Cardiología y Cirugía Cardiovascular Infantil. Artaza 0 y Zilleruelo R. Ed. Mediterráneo. Santiago, Chile. 1989: 75-77.

7. Catañedo AR, Freed MD. Willians RG. Norwood W: Repair of tetrakgy of Fallot in infancy. I Thorac Cardiovase Surg 1977; 74: 372-381.

8. Hudspeth AJ, Cordell AR, Johnston FR: Transatrial approach to tatal correction of tetralogy of Fallot. Circulation 1963; 27: 796-800.

9. Harken AH, Horowitz L.N, Josephson ME: Surgical correction of recurrent sustained ventricular tachycardia following complete repair of tetralosy of Fallot. J Thorac Cardiovase Surg 1980; 80: 779.781.

10. Kowashina $Y$, Matsuda $H$. Hirose $H$, Nakano $S$. Shirakuro $R$, Kohayashi $J$ : Ninety consecutive corrective operations for tetralogy of Fallot with or without minimal right ventriculotomy. J Thorac Cardiovasc Surg 1985:90: 856-863.

11. Mc Grarh LB, González-Lavin L: Determination of the need for a ventriculotomy in the repair of tetralogy of Fallot. I Thorac Cardiovasc Surg 1988; 96: 947-951.

12. Perrotit H, Drblik SP, Montigny M: Comparison of cardioyascular adjustments to cxercise in adolescents 8 to 15 years of age after correction of tetralogy of Falloz, ventricular septal defect or atrial septal defect. Am J Cardiol 1989; 64: 213217.

13. Nakata $S$, Imai $Y$. Takanoshi $Y$ : A new method for the quantitative standardization of cross-sectional areas of the pulmonary atteries in congenital heart diseases with decreased pulmonary blood flow. J. Thorac Cardinvasc Surg 1984; 88: 610-619.

14. Kirklin JW, Blackstone $E H$, Kirklin JK, Pacifico $A D$ : Surgical results and protocols in spectrum of tetrilogy of Fallot. Ann Surg 1983; 198: 251265 .

15. McCaughon BC, Danielson GX. Driscoll DJ, MCGoon DC: Tetralogy of Fallot with absent putmonary valve. I Thorac Cardiovase Surg 1985 ; $89: 280-287$.

16. Dietl $C H$, Torres A, Cazzaniga $4 \%$ Right atrial approach for surgical correction of tetratogy of Fallot. Ann Thorac Surg 1989; 48: 546-552.

17. Kirklin $J W$ : Ventricular scptal defect and pulmonary stenosis or atresia. In. Cardiac Surgery. Shon Willey \& Sons. New York 1986; pp. 699 . 819.

18. Kirklin JW, Blackstone EH, Calvin EV, McConnell ME: Early primary correction of tetralogy of Fallot. Ann Thorac Surg 1988; 45: 231-233.

19. Kirklin $J K$, Kirklin $J \boldsymbol{W}$. Blackstone $E H$, Mitano $A$, Pacifico $A D$ : Effect of transanular patching on outcome after repait of tetralogy of Fallot. Ann Thorac Surg 1989; 48: 783-791.

20. Clarke DR, Camphell DN. Pappas G: Pulmonary allograft condujt Jepair of tetralogy of Fallot (An alternative to transanularn patch repair) J Thorac Cardiovase Surg 1989; 98 : 730-737.

21. Arciniegas E, Farooki ZQ. Hakimi M, Perry BI, Green EW: Early and late results of total correc. tion of tetralogy of Fallot. J Thorac Cardiovasc Surg 1980;80;770-778.

22. Fuster $V$, McGoon $D C$, Kentedy MA, Ritter $D G$, Kirklin JW: Long tem evaluation (12-22 years) of open heart surgery for tetsalogy of Falbot. Ann J Cardiol 1980:46:635-642.

23. Horneffer PJ, Zahka KG, Rowe $S A$, Manolio $T A$, Gott $V L$, Reitz BA; Long term results of total repair of tetralogy of Fallot in Chilhood. Ann Thorac Surg 1990;50: 178-189

24. Artaza O: Postoperatorio. En Manual de Cardiología y Cirugía cardiovascular infantil. Artaza O y Zilleruelo R. Ed. Mediterraneo. Santiago, Chile, 1989: $259-266$.

\section{AVISO A LOS AUTORES}

Por acuerdo del Comité Editorial, la Revista Chilena de Pediatria devolverá sin tramitar todos los trabajos que no den estricto cumplimiento a) Reglamento de Publicaciones, que se edita en cada número, y a las Instrucciones a los Autores, que aparecen en los números 3 y 6 de cada volumen desde 1986. 